\title{
A Case Study in Strategic Sustainability: British Petroleum (BP) Company
}

\author{
Shengji Li, Jinxia Zhu, Kangyin Lu \\ 123School of Business, Northeast Normal University, Changchun, China \\ lishengji1986@sina.com, zhuzhu.8175@163.com, luky440@nenu.edu.cn
}

\begin{abstract}
British Petroleum (BP) Company, generally regarded as $\mathrm{BP}$, is one of the world's distinguished international oil and gas companies. BP specializes in making and selling useful products which are generated from oil and natural gas. This report begins with brief overview and sustainability situation of BP. After that, the particular progress towards sustainability achieved at BP is analysed and critically evaluated through sustainability change matrix. On the basis of stressing its sustainability position, general direction of sustainability change process could be shaped. During the change process, influential factors for sustainability change progress in BP will be listed. This is followed by recommendations put forward accordingly. Finally, this section embraces change strategies and specific sustainability actions in BP as well. In order to reach the strategic position, sustainability programs are designed and presented step by step.
\end{abstract}

Index Terms - British Petroleum (BP) Company, Strategic Sustainability, Sustainability Programs

\section{BP Company and Its Sustainability Situation}

British Petroleum (BP) Company, generally regarded as $\mathrm{BP}$, is established in 1909 after oil found in a rugged part of Persia. The corporate headquarter is in London, England. As one of the world's distinguished international oil and gas companies, BP specializes in making and selling useful products which are generated from oil and natural gas. It concentrates on offering fuel for transportation, energy for heat and light, retail services and petrochemicals products for everyday items. The scope of business in BP spreads across six continents, and more than 80 countries are accessible for the products and services.

With the popularization of industrialization, the emissions of carbon dioxide and temperature of the earth's surface are increasing gradually. In view of this status, BP is conscious of promptly taking measures for integrating ecological, social and economical sustainability. According to Browne (1997), petroleum occupies 43 percent of the total $\mathrm{CO} 2$ emissions caused by burning fossil fuels. It is urgent for $\mathrm{BP}$ to monitor and control its own $\mathrm{CO} 2$ commissions. Meantime, energy efficiency standards are generated and effective implementation of cost-effective technology is advocated. In order to design the right technology for restricting greenhouse gas emissions, BP also collaborated with Greenhouse Gas Programme of the International Energy Agency. Kolk (2008) stated that BP innovatively embarked on expanding solar energy technology with concrete investment and capacity plans since 1997. From then on, BP keeps working on the research and development of corporate sustainability. The characteristics of these activities have in common are the combination of environmental protection, economic development, social progress and considering for the future.

Bulkin (2003) argued that sustainability can be nurtured through undertaking corporate social responsibility (CSR). This is the beginning of BP's action on publicly assuming CSR and the sign of BP Company was changed into green flowery design. It is costly for BP to make CSR efforts which is why long-standing CSR debate exists within company. In the light of Hicks (2010), the more profits in BP, the more funds allocated to CSR efforts. For this reason, CSR is a double-edged sword and a well-crafted CSR plan is needed in BP.

In the oil industry, BP is famous for its sustainability reports with clear structure, intelligible words and detailed information in terms of its sustainability progress and impacts on the company as well as the whole society. BP has already worked with other Non-Governmental Organizations (NGO) and negotiated with communities in a long-term (Yan, Xiao \& Sha 2009). Due to the pre-existing sustainability achievement and remarkable enhancement of $\mathrm{BP}$ in greenhouse gas emissions (GHG), BP is evaluated as greenest oil company by Greenopia. However, marked by the accident in the Gulf of Mexico on 20 April 2010, the corporate sustainability of BP fell into worst question. The accident led to a large area of oil spill and damaged worldwide trust in BP (Business and the Environment 2010).

\section{Sustainability Analysis of BP}

During the development of corporate sustainability, six phases are generated in the light of different scales in human sustainability (HS) and ecological sustainability (ES). As mentioned by Dunphy, Griffiths and Benn (2003), these six phases are rejection, non-responsiveness, compliance, efficiency, strategic proactivity and the sustaining corporation. The characteristics of strategic proactivity phase can be described from two dimensions: HS and ES. In human sustainability, diversified and mixed workforce structure is advocated. The company is more inclined to recruit the most talented employees and develop intellectual and social capital. Besides, product and service innovation is placed on the top position for meeting emerging market demands. Aimed at strengthening communities' cohesion, company focuses on investing and involving itself into the reconstruction of communities. For ecological sustainability, environmentally damaging products are replaced by renewable and environmentally friendly ones.

Reasonably, BP should be positioned into strategic 
proactivity phase in the process of recovering from Gulf of Mexico oil spill. It can be revealed from 'BP in figures' that the percentage of people from UK and US racial minorities in group leadership increased from 5\% in 2006 to $7 \%$ in 2010. People from beyond the UK and US in group leadership occupied $19 \%$ in 2011 which was close to $20 \%$ in 2006 . Moreover, contracts terminated or not renewed due to noncompliance or unethical behaviour decreased heavily from 69 in 2006 to 14 in 2011. These figures are the good evidence of proving the diversified workforce structure and appropriate employee recruitment. For the sake of identifying, monitoring and managing risk, BP put more emphasis on introducing safety and operational risk (S\&OR) function and restructuring upstream business. As mentioned by Benn, Dunphy and Perrott (2011), BP made investments on facilitating the region recover because of the negative impact on its communities. With the help of stressing code of conduct, BP also made great efforts on consolidating safety culture and relationships with contractors and stakeholders. Due to the increased amount of carbon dioxide and greenhouse gas emissions, BP focused on researching low-carbon energy with high security and affordability. In this regard, it can be concluded that BP contributes a lot to strategic sustainability via enhancing both ecological and human capabilities. Overall, BP is a good practitioner of reaching triple bottom line approach. To a certain extent, the sustainable development of BP was achieved through integrating ecological, social and economic sustainability.

In spite of great sustainability progress, a number of sustainability problems still need to be noticed in BP. Firstly, BP did not pay more attention on improving ecological sustainability until the accident happened. Instead, BP concerned more about financial and technological factors which occupied hugely in business strategies. What BP did for the accident was more likely about risk-reduction exercise other than derived from its initiative intention. The things BP did in this stage can be classified into compliance phase in the development of corporate sustainability. Furthermore, at the beginning, BP underlined cost efficiencies by means of eliminating waste in ecological issues. Nevertheless, a few environmental issues were easily ignored because they might not have adverse impact on the company temporarily. After the accident, not only was BP trying to adopt risk-reduction measures, but also it developed its sustainability from efficiency phase to strategic proactivity phase. BP made some evolutionary paths on the basis of obtaining great sustainable progress; the sustainability status of BP can be positioned in sustainability change matrix (quadrant 4 and 5).

In order to be evolved into the sustainable corporation, the implementation of sustainability change process is of great importance to BP. Firstly, as regarded as the internal core capabilities in BP, developing low-carbon energy should be continued substantially. Secondly, with the aim of internally stimulating cultural change, BP has the responsibility to renew and enhance the values and behaviors which are good for sustainable development. With the enhancement of employee awareness, the change process will be prompted internally
(Thompson 2008). Besides, James-Overheu, Christina \& Julia (2009) stated that the decent recruitment and training system are good for human sustainability through shaping into the knowledge-based organization. What's more, external parties and organizations play an essential part in driving the sustainability change process. As the delegation of shareholders, the board in BP is accountable for the oversight of risk and monitoring the performance of the business. Based on the gain of multilateral feedback on how BP performs, stakeholders have the right to give advice on sustainable development. Hawkins (2006, pp. 145) claimed that stakeholders include customers, regulators, suppliers, NonGovernmental Organizations (NGOs), consumers, employees and investors. Analysis of organizational corporation and sustainable issues management had been generated by some investment managers and investment banks. If BP did not make risk management, the cost of non-compliance would be huge. Safety, ethics and environment assurance committee is capable of monitoring the management of non-financial risk. Gulf of Mexico committee is in charge of providing and recording the delivery of restoration in that area. The positive attitude of BP on its own involvement in communities is beneficial for sustainability change process. In terms of business activities, BP usually collaborates with suppliers and partners with the consideration of consistent code of conduct and compliance with legal requirements. In the recruitment stage, BP is more inclined to hire people with specialist skills and great potential to work for the company. The concrete diversity and inclusion (D\&I) plans and clear no-tolerance policy with regard to abusive behaviours in BP boost the application of sustainable development internally (MarJ 2008). BP also has form the culture of innovation which plays an indispensable part in boosting incremental change process. In view of emerging demand for renewable energy, BP has been concentrating on exploring low-carbon energy even with huge cost. Schaltegger, Burritt and Petersen (2003) stated that improving the efficiency of production in innovation of product is very necessary. Huge research and development (R\&D) cost and two many suggestions might hinder the sustainability change process in BP.

\section{Recommendations}

Based on the above analysis, for moving effectively from strategic proactivity to the sustaining corporation, a series of concrete sustainability actions in BP are recommended as following. According to Dunphy, Griffiths and Benn (2003), eight steps of incremental change can be concluded along with the specific explanation.

Step 1: the clear vision and strategic goals deserve first priority in changing BP. Specifically, the vision of BP can be characterised by growing value for shareholders in a safe and sustainable way. With the aim of integrating ecological, social and economic sustainability appropriately, strategic goals should be set reasonably. For instance, safety working process, comprehensive risk management, qualified employees, innovative culture and good relationships with partners and suppliers and so on. 
Step 2: current sustainability situation in BP needs to be evaluated. There is a profound connection between Deepwater Horizon accident and detrimental impact on life along the Gulf Coast, ranging from environmental and wildlife to economic and social issues. The immediate response activities of BP towards this accident, long-term cooperation with business partners, growing support from shareholders and excellent company management are the enablers for making BP more sustainable. The barriers are the less initiatives of BP in conducting its business activities prior to the accident. Besides, in terms of improving sustainability operations in BP, too many suggestions might hinder the direction. Consultant group in BP should do more work on assessing several options and maximally considering most shareholders' interest.

Step 3: the type of change programmes BP needed should be assessed. S\&OR monitor system is needed for complying operations with BP's operating management system. With the ingredients of standards, processes, tools and methodologies, risk management system is also necessary for BP to figure out, monitor and manage risk. New performance management system is urgent to be established as a result of its effective relation to safety and reward, and links with values and behaviours. In order to recruit the qualified employees, explicit recruitment and training system should be promoted in BP (Heincke 2006). In particular, Gulf of Mexico committee should in charge of recording and developing restoration acticities.

Step 4: change agents could be identified in BP. A team of constant group should be professional and knowledgeable in regard to BP's sustainability history and situation. After gathering the information of BP's sustainability, consultant group has the responsibility to produce a template for action plan and time line. For instance, "innovation board" can be suggested for recording business transformation, top-line revenue growth, cost reductions and health, safety and environment (Brown \& Markham 2007).
Step 5: new practices and innovation could be piloted. Operational efficiency of risk management system in BP can be tested. Once confirming its efficiency, BP can make good promotion across the company even introducing it to other corporations.

Step 6: list further resources for new programs in BP. Relevant resources need to be updated after confirming the efficiency of sustainability programs. Time, money, People and management support ought to be put into practice.

Step 7: communicate and extend the program. BP is accountable for informing the whole organization about the application of sustainability programs. BP plays a significant role in coordinating and facilitating the change process.

Step8: align organization systems. When sustainability programs are determined to be used in truly organizational dynamics, operating systems, reward systems, information systems and organizational structures should be adjusted and modified accordingly.

\section{References}

[1] Brown, J 1997, 'Positive action by industry needed on global climate change, BP says', Oil \& Gas Journal, vol. 95, no.23, pp. 14-19.

[2] Brown, C \& Markham, S 2007, 'Innovation learning at BP', Research Technology Management, pp. 9-14.

[3] BP PLC2010, 'BP named greenest oil company', Business and the Environment, 03 May, p. 9-10, viewed 23 October 2011,

[4] Bulkin, BJ 2003, 'BP=bringing profits: In a socially responsible way', Mid-American Journal of Business, vol. 18, no. 1, pp. 7-10.

[5] Benn, S, Dunphy, D \& Perrott, B 2011, Case in Corporate Sustainability and Change: A Multidisciplinary Approach, $1^{\text {st }}$ edn, Tilde University Press, Australia.

[6] Dunphy, D, Griffiths, A \& Benn, S 2003, Organizational Change for Corporate Sustainability: a guide for leaders and change agents of the future, 2nd edn. Routledge, London \& New York.

[7] Ernst \& Yong LLP 2011, BP Sustainability Review, London, viewed 23 October 2011,

[8] Ernst \& Yong LLP 2011, BP Sustainability Reporting 2010 Gulf of Mexico restoration, London, viewed 22 October 2011,

[9] Hicks, MJ 2010, 'BP: Social Responsibility and the Easy Life of the Monopolist', American Journal of Business, vol. 25, no. 2, pp. 9-10. 